\title{
Using Traffic Flow for Cluster Formation in Vehicular Ad-hoc Networks
}

\author{
Mohammad S. Almalag \\ Department of Computer Science \\ Old Dominion University \\ Norfolk, VA 23529 \\ Email: malmalag@cs.odu.edu
}

\author{
Michele C. Weigle \\ Department of Computer Science \\ Old Dominion University \\ Norfolk, VA 23529 \\ Email: mweigle@cs.odu.edu
}

\begin{abstract}
We present a lane-based clustering algorithm designed to provide stability in cluster lifetime for vehicular adhoc networks (VANETs) in urban scenarios. Stable clustering methods reduce the overhead of re-clustering and lead to an efficient hierarchical network topology. During the creation of VANET clusters, cluster members select one member to be the clusterhead. Fewer clusterhead changes result in a more stable cluster. To achieve this goal, cluster members must select a member that has the potential to be a clusterhead longer than other cluster members. Our method aims to select a clusterhead based on the lane where most of the traffic will flow.
\end{abstract}

\section{INTRODUCTION}

Vehicular Ad Hoc Networks (VANETs) are considered to be a class of Mobile Ad Hoc Networks (MANETs); they share the same underlying philosophy. In the US, VANETs use 75 $\mathrm{MHz}$ of spectrum in the 5.850 to $5.925 \mathrm{GHz}$ band specially allocated by the U.S. Federal Communications Commission for Vehicle-to-Vehicle communication (V2V) and Vehicle-toInfrastructure communication (V2I) using Dedicated ShortRange Communication (DSRC) technology [19]. The main goal of VANETs is to share information such as traffic data and road conditions to increase the safety of drivers.

Even though VANETs are considered to be a class of MANETs, they have a number of specific characteristics that make many solutions for general MANETs unsuitable for VANETs. Some of the VANET characteristics are a high number of nodes, high node mobility, frequently changing network topology, network disconnection caused by the partitioning of the network, driver behavior and the anticipated mobility of nodes. However, VANETs have some advantages over MANETs. Nodes in VANETs have more processing power and storage space and typically have no battery limitations.

As an ad hoc network, a VANET cannot rely on specialized hardware for infrastructure to support network stability. Each node in a VANET is required to maintain its own connectivity to other nodes in the network. With the large number of nodes and the lack of routers, a flat routing scheme, where each node acts as a router, may cause serious scalability and hidden terminal problems. One possible solution to these problems is hierarchical clustering. In addition, using clustering can lead to more node coordination and fewer nodes interfering with each other.
A cluster is a group of nodes that can communicate without disconnection and that identify themselves to be part of a cluster. These nodes select a clusterhead to coordinate the communication among them. Clustering in VANETs requires selecting a clusterhead that results in a stable cluster. This process is carried out by each node broadcasting its information to all other neighboring nodes. After the nodes have decided on the clusterhead, the clusterhead will be able to communicate directly to all other cluster members and may act as the relay node of communications to other cluster members and other nodes in different clusters. Significant time and channel bandwidth will be consumed to complete this process.

A considerable amount of work has gone into the development of clusterhead selection algorithms that would be suitable to form highly stable clusters [20], [12]. All the algorithms are trying to minimize cluster reconfiguration and clusterhead changes, which are unavoidable due to the dynamic nature of the network. Having a good clustering algorithm requires selecting the clusterhead that will serve most of the vehicles for the longest possible time. Knowing the traffic flow and the general information of a vehicle, such as speed, direction, location and lane, should lead to better clusterhead selection.

In this paper, we present a new clustering algorithm whose objective is to extend the lifetime of a clusterhead. We take advantage of knowing the exact lane of vehicles on the road and then broadcast this knowledge to other nearby vehicles to determine the optimal clusterhead. Our method of selecting the clusterhead is the key to achieving a more stable cluster. We compare our selection of the clusterhead with three other approaches: Lowest-ID [11], Highest-Degree [16] and the Utility Function [6].

This paper is organized as follows. Section II reviews background and related work. Section III presents our approach and the elements that will be measured. Section IV presents our simulation and the scenario setup, followed in Section V by performance evaluation. Finally, Section VI offers concluding remarks.

\section{BACKGROUND AND RELATED WORK}

A cluster is conceptual structure where a group of nodes, cluster members, identify themselves to be part of cluster. The node designated to be the clusterhead will broadcast packets 
to other nodes in the cluster. All cluster members are within one-hop communications range of the clusterhead, thus the potential cluster size increases with the transmission range. Each clusterhead may act as a relay point for communication between the cluster's members [7]. Some clustering schemes assign another node to act as a relay node for communication with other clusters [20], as shown in Fig. 1.

This research focuses on the process of selecting the clusterhead that will last the longest. We ran our experiments considering one cluster traveling through two intersections under different clusterhead selection algorithms.

In the remainder of this section, we will describe work on lane detection and provide an overview of the LowestID, Highest-Degree and utility function clustering algorithms which we will compare our work against.

\section{A. Lane Detection}

Our proposed algorithm is based on the assumption that each vehicle knows its exact lane on the road via a lane detection system and an in-depth digital street map that includes lane information, such as NAVTEQ's NAVSTREETS [14]. A lane detection system is an important element of many applications in VANETs, such the Extended Emergency Brake Light system [9].

The Global Positioning System (GPS) is the primary system that is used for vehicle localization. However, GPS has weaknesses when it comes to updating the positioning data and when there is no signal. GPS has a $5 \mathrm{~m}$ error which is larger than the distance between lanes. There has been much research on detecting and localizing lanes on roads. Several algorithms have been proposed using different techniques. Some methods use GPS combined with a wheel odometer [4], which provides relative localization as it detects changes in pose relative to the previous pose. The advantage of the wheel odometer is that it is high resolution and simple to use. It can typically detect movements on the order of tenths of millimeters. Other algorithms do not use GPS, and instead use techniques such as vision [2], [3], [15], LIDAR (Light Detection and Ranging) [10] and a beacon network using infrastructure to triangulate vehicle position [13].

\section{B. Clustering Algorithms}

Here we present the three clusterhead selection algorithms that we will be comparing our work against.

1) Lowest-ID: The Lowest-ID clustering algorithm [11] is based on selecting the clusterhead with the lowest ID, where each node has a fixed ID. Simply, each node broadcasts its ID to other nodes in range. When a node receives the messages from other nodes, it determines the clusterhead as the node with lowest ID. This algorithm is very simple and stable for general MANET applications. However, in VANETs, the lowest ID clusterhead is not always the ideal selection because the movement of the vehicles is not considered.

2) Highest-Degree: The Highest-Degree algorithm [16] selects the clusterhead based on the node connectivity to the other nodes in the same cluster. Each node knows the number

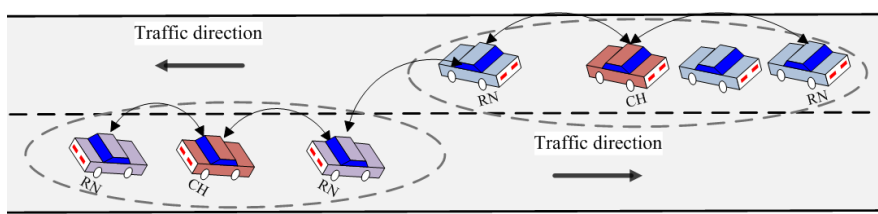

Fig. 1. Two clusters communicating with each other through relay nodes $(\mathrm{RN})$ that are assigned by the clusterhead $(\mathrm{CH})$ of each cluster.

of other nodes in range and then broadcasts this knowledge to the others. The node with the maximum number of neighbors is selected as the clusterhead. This algorithm is one of the basic techniques for clusterhead formation in MANETs. However, it is not stable for VANETs due to the nature of the nodes' movement. If the clusterhead changes its behavior at any moment, the connectivity level could change dramatically.

3) Utility Function: The Utility Function algorithm [6] of clusterhead selection in VANETs performs better than the previous two algorithms, Lowest-ID and Highest-Degree. This algorithm is based on a multiple-metric weighting algorithm. In the process of the clusterhead selection, the closest position to the average and the closest velocity to the average of all proximal vehicles are calculated along with connectivity level to determine the most stable clusterhead. Periodically, each node broadcasts its status to other nodes in range. When the node receives this information, it starts to evaluate each node by using the utility function. The node with the highest value is chosen to be the clusterhead.

In a highway environment, this algorithm has been shown to provide better results than the classic MANET algorithms. It puts the position and velocity, which are major VANET characteristics, into consideration. However, it still ignores the traffic flow on the road. For example, in an urban scenario where are many intersections, if the clusterhead is located on the leftmost lane, it has to turn left even if most of the vehicles are going straight. In this case, the vehicles will need to perform the process of clusterhead selection again.

\section{APPROACH}

We considered urban scenarios with intersections in the design of our proposed algorithm. The clusterhead will be selected based on the flow of the majority of traffic. For example, if the road has four lanes and three of them are going straight, the clusterhead should be selected from the lanes that are going straight. This research applies the knowledge of each vehicle's lane and the flow direction of each lane.

In urban scenarios, traffic flow splits at each intersection. There are three main traffic flows at an intersection: Left Turn (LT), Right Turn (RT), and No Turn (NT). The intersection may have all three types of traffic flows or only some of them. LT is applied to the leftmost lane(s) if it splits the traffic to the left, RT is applied to the rightmost lane(s) if it splits the traffic to right, while NT is applied to the lane(s) in the middle if traffic goes straight.

Our proposal follows the same general idea as the Utility Function [6], but employs a different set of rules. We consid- 
ered the effect of traffic flow, using lane information, on the process of clusterhead selection. Each vehicle computes and broadcasts its Clusterhead Level ( $C H L)$ along with its speed, position, etc. The vehicle with the highest $C H L$ will be selected as the clusterhead. If the selected clusterhead is a member of another cluster, the nodes will choose the second highest, etc. CHL is defined as

$$
C H L_{i}=N C L(t)_{i}+A D L_{i}+A V L_{i}
$$

where NCL is the network connectivity level, ADL is average distance level, and AVL is average velocity level. The computation of each of these metrics is described below.

\section{A. Lane Weight}

The key to our approach is to consider the lane a vehicle belongs to. We apply to each metric a lane weight $(L W)$ for each traffic flow (LT, RT and NT). The weight is determined based on the total number of lanes on the roadway $(T N L)$ and the number of lanes for each traffic flow (NLTF). If the road has three different traffic flows, we will have three different $L W s . L W$ is defined as

$$
L W_{k}=\frac{1}{T N L} \times N L T F_{k}
$$

where $k$ is the lane number. For example, if we have a road of four lanes where one lane is $L T$, one lane is $R T$, and two lanes are $N T$, then the $L W$ for each traffic flow will be $L W_{L T}$ $=L W_{R T}=0.25$ and $L W_{N T}=0.50$. If a vehicle is on a lane with traffic flow $L T$, then it will use $L W_{L T}$. In the equations that follow, $L W_{T F}$ represents the $L W$ for the traffic flow of the vehicle performing the computation.

\section{B. Network Connectivity Level}

To compute the Network Connectivity Level (NCL), we need to calculate the overall NCL and the NCL for each traffic flow. The overall NCL, $\alpha$, is the maximum number of vehicles that are directly connected to vehicle $i$. This is defined as

$$
\alpha_{i}(t)=\sum_{j} A(i, j, t)
$$

where $j$ is a potential neighboring vehicle. $A(i, j, t)$ is equal to 1 if a connection between $i$ and $j$ exists at time $t$, and is equal to 0 otherwise. At this point, we have calculated the connectivity level between a vehicle and all other vehicles on the road. Now, we calculate the connectivity level for a vehicle and the vehicles in the traffic flow it belongs to. The traffic flow connectivity level $\beta$ for vehicle $i$ is defined as

$$
\beta_{i}(t)=\sum_{j_{T F}} A\left(i, j_{T F}, t\right)
$$

where $j_{T F}$ is a vehicle in the same traffic flow as vehicle $i$. After calculating both levels of network connectivity, we define the total connectivity level for vehicle $i$ on a lane belonging to traffic flow $T F$ as

$$
N C L_{i}(t)=\beta_{i}(t)+\alpha_{i}(t) \times L W_{T F}
$$

where $L W_{T F}$ is the lane weight for the lane that vehicle $i$ occupies.

\section{Average Distance Level}

To calculate the Average Distance Level (ADL), we calculate the overall average absolute distance, $\delta_{i}$ between vehicles that are directly connected to vehicle $i$. This is defined as

$$
\delta_{i}=\frac{\sum_{j} \sqrt{\left(x_{j}-x_{i}\right)^{2}+\left(y_{j}-j_{i}\right)^{2}}}{N V}
$$

where $j$ is any vehicle connected to $i$, and $N V$ is the total number of vehicles that are directly connected to $i$ in any lane.

Next, we calculate the average absolute distance, $\chi_{i}$, between vehicle $i$ and other vehicles in the same traffic flow, $T F$. This is defined as

$$
\chi_{i}=\frac{\sum_{j_{T F}} \sqrt{\left(x_{j}-x_{i}\right)^{2}+\left(y_{j}-j_{i}\right)^{2}}}{N V_{T F}}
$$

where $j$ is any vehicle in the same traffic flow and connected directly to $i$, and $N V_{T F}$ is the total number of vehicles that are directly connected to $i$ and in the same traffic flow.

The ADL for vehicle $i$ in traffic flow $T F$ is defined as

$$
A D L_{i}=\chi_{i}+\delta_{i} \times L W_{T F}
$$

\section{Average Velocity Level}

We calculated the overall Average Velocity Level (AVL) as the difference between the average velocities of all vehicles in range and the candidate clusterhead velocity. Then, we add this to the product of $L W$ and the average velocity for the traffic flow. The overall AVL, $\sigma_{i}$, for vehicle $i$ is defined as

$$
\sigma_{i}=\sum_{j}\left|V e l_{i}-V e l_{j}\right|
$$

where $j$ is a potential neighboring vehicle, and $V e l_{i}$ is the velocity of vehicle $i$.

Now, we calculate the AVL, $\rho_{i}$, for vehicle $i$ and the traffic flow it belongs to. This is defined as

$$
\rho_{i}=\sum_{j_{T F}}\left|V e l_{i}-V e l_{j}\right|
$$

where $j_{T F}$ is a vehicle in the same traffic flow as vehicle $i$.

The AVL for vehicle $i$ in traffic flow $T F$ is defined as

$$
\begin{gathered}
A V L_{i}=\rho_{i}+\sigma_{i} \times L W_{T F} \\
\text { IV. SIMULATION }
\end{gathered}
$$

\section{SIMULATION}

Our proposed clusterhead selection algorithm was evaluated through detailed simulation. We used the ns-3 network simulator [8], which is a follow-on to the popular ns-2 simulator. For VANETs, we used modules [1] that added well-known traffic mobility models, the Intelligent Driver Model (IDM) [18] and the MOBIL lane change model [17].

The scenarios shown in Figs. 2 and 3 were implemented for two intersections of Hampton Boulevard in Norfolk, VA. The length of the road is set to $3 \mathrm{~km}$. The intersections split the traffic into three different directions. The first intersection (Fig. 2) has five lanes, dividing the traffic into three directions: two lanes to the left, two lanes going straight and one lane going right or straight. The second intersection (Fig. 3) has 


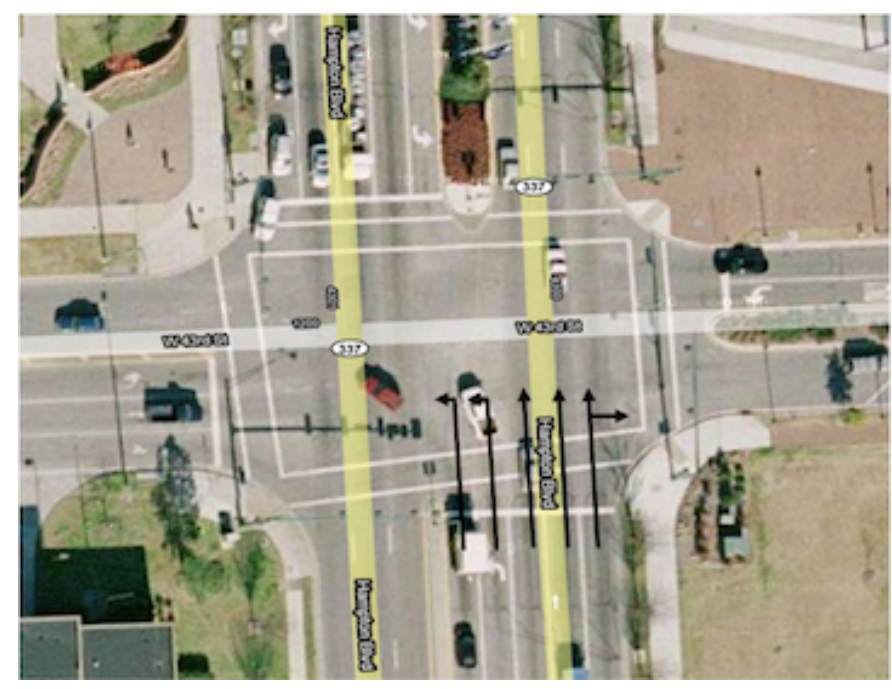

Fig. 2. Intersection of Hampton Blvd. and 43th Street. It shows 5 lanes, 2 areturning left, 2 are going straight and 1 lane is going right/straight.

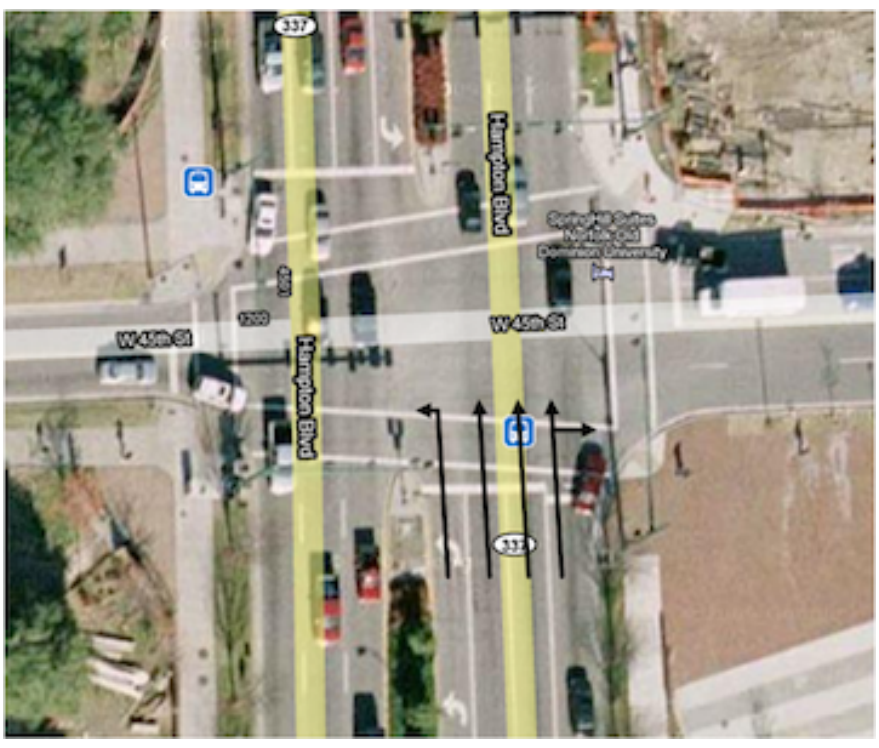

Fig. 3. Intersection of Hampton Blvd. and 45th Street. It shows 4 lanes, 1 isturning left, 2 are going straight and 1 lane is going right/straight.

four lanes, dividing the traffic into three directions: one lane to the left, two lanes going straight and one lane going right or straight. We focused only on one traffic direction, since oncoming traffic is not part of the studied cluster. The clusterhead that will be selected by our algorithm will be towards the middle of the cluster and continuing in the same direction as the majority of the traffic flow, as shown in Fig. 4. The traffic density we used for our simulation was a medium level (60 vehicles per lane per $\mathrm{km}$ ), averaging a $10 \mathrm{~m}$ gap between vehicles. The vehicle type ratio used for this simulation was $20 \%$ trucks and $80 \%$ sedans. We inject vehicles on the road for the first 120 seconds of simulation time, and then we follow the vehicles until they leave the road. We ran 10 different runs for each scenario.
TABLE I

SCENARIOS TESTED DURING THE SIMULATION

\begin{tabular}{ccc}
\hline \hline Scenario & Transmission Range & Max Speed Limit \\
\hline 1 & $100 \mathrm{~m}-150 \mathrm{~m}$ & $40 \mathrm{~km} / \mathrm{h}$ \\
2 & $100 \mathrm{~m}-150 \mathrm{~m}$ & $80 \mathrm{~km} / \mathrm{h}$ \\
3 & $100 \mathrm{~m}-150 \mathrm{~m}$ & $120 \mathrm{~km} / \mathrm{h}$ \\
4 & $150 \mathrm{~m}-200 \mathrm{~m}$ & $40 \mathrm{~km} / \mathrm{h}$ \\
5 & $150 \mathrm{~m}-200 \mathrm{~m}$ & $80 \mathrm{~km} / \mathrm{h}$ \\
6 & $150 \mathrm{~m}-200 \mathrm{~m}$ & $120 \mathrm{~km} / \mathrm{h}$ \\
7 & $200 \mathrm{~m}-250 \mathrm{~m}$ & $40 \mathrm{~km} / \mathrm{h}$ \\
8 & $200 \mathrm{~m}-250 \mathrm{~m}$ & $80 \mathrm{~km} / \mathrm{h}$ \\
9 & $200 \mathrm{~m}-250 \mathrm{~m}$ & $120 \mathrm{~km} / \mathrm{h}$ \\
10 & $250 \mathrm{~m}-300 \mathrm{~m}$ & $40 \mathrm{~km} / \mathrm{h}$ \\
11 & $250 \mathrm{~m}-300 \mathrm{~m}$ & $80 \mathrm{~km} / \mathrm{h}$ \\
12 & $250 \mathrm{~m}-300 \mathrm{~m}$ & $120 \mathrm{~km} / \mathrm{h}$ \\
\hline
\end{tabular}

We test the stability of the cluster by counting the number of clusterhead changes. Before each intersection, we use clusterhead selection algorithm to choose the clusterhead and observe if the clusterhead changes for the majority of traffic after the intersection.

We varied the transmission range and speed limit to test the stability of the clusterhead. Different transmission ranges and speeds will affect the clusterhead selection when connectivity is part of the clusterhead selection algorithm. All of our scenarios are listed in Table I.

With our proposed scheme, a vehicle sends its calculated $C H L$ along with the general traffic data using periodic beacons. The cluster formation will be processed every 20 seconds.

Both MANET clustering algorithms we discussed earlier were simulated, as well as the VANET Utility Function algorithm, which was implemented as explained in [5].

\section{RESULTS}

The simulation results show the performance of the different clustering algorithms, Lowest-ID, Highest-Degree, Utility Function and our traffic flow algorithm under different wireless transmission ranges (100-300 meters) and different maximum vehicle speeds (40-120 kilometers/hour). For all other algorithms besides ours, the clusterhead selected was not always continuing in the same traffic flow as most of the vehicles.

Fig. 5 summarizes the effect of transmission range on the clusterhead selection at the first intersection with the speed limit of $40 \mathrm{~km} / \mathrm{h}$. We recorded if the clusterhead changed after the intersection. The graph shows the number of clusterhead changes summed over all 10 runs. The first intersection has

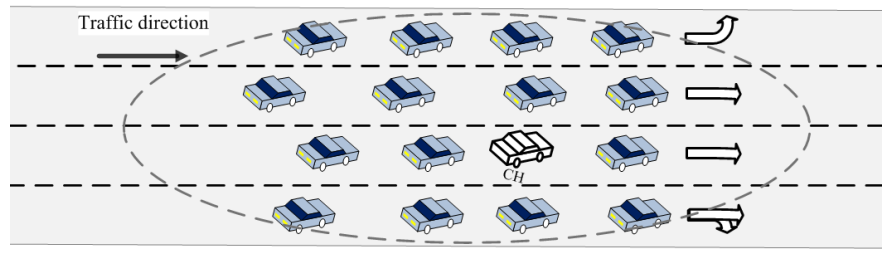

Fig. 4. One cluster showing the position of the clusterhead $(\mathbf{C H})$ in the middle. The majority of traffic is going straight and the clusterhead is following that. 


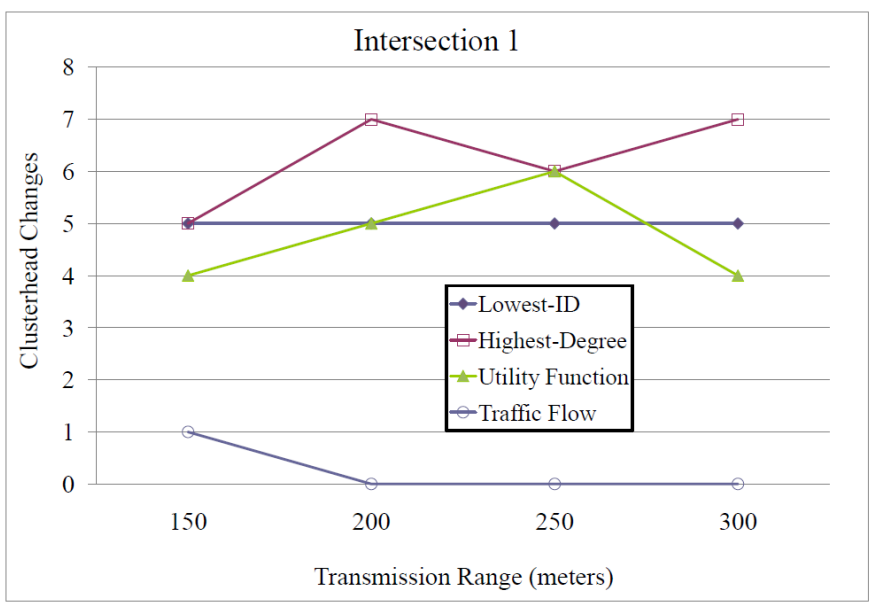

Fig. 5. Clusterhead changes vs. Transmission Range at the first intersection, where we have 5 lanes with different traffic flows. The maximum speed is $40 \mathrm{~km} / \mathrm{h}(25 \mathrm{mph})$

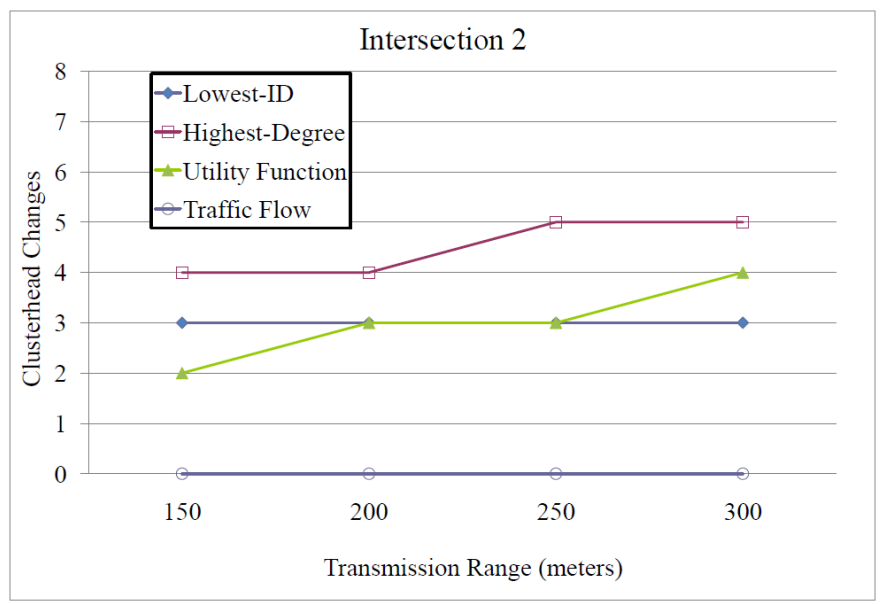

Fig. 6. Clusterhead changes vs. Transmission Range at the second intersection, where we have 4 lanes with different traffic flows. The maximum speed is $40 \mathrm{~km} / \mathrm{h}(25 \mathrm{mph})$

five lanes, two lanes turning left, two lanes going straight and one lane turning right or staying straight.

Notably, our algorithm performed better than the other algorithms because everytime the clusterhead is selected, it belongs to one of the lanes where most of the traffic is flowing in its direction. In one case using our algorithm, the clusterhead changed because the selected clusterhead was in one of the lanes where the traffic turned. The reason for that is the clusterhead changed lanes to the left turn lane after the clusterhead procedure was performed. For the Lowest-ID algorithm, the transmission range does not have any impact on the selection of the clusterhead, so its clusterhead selections were the same with all of the transmission ranges that we tested. As expected, the Highest-Degree and the Utility Function algorithms performed differently when the transmission range changed. Transmission range affects the number of nodes that are connected to the clusterhead, and both of these algorithms use degree of connectivity when choosing a clusterhead.
Fig. 6 shows the effect of transmission range on the clusterhead selection at the second intersection with the speed limit of $40 \mathrm{~km} / \mathrm{h}$. Our algorithm performed perfectly compared to all other algorithms. The reason for that is the lanes at this intersection are fewer than the first one, which means less traffic is going in a different direction than straight.

We show only results for the speed limit of $40 \mathrm{~km} / \mathrm{h}$ as changing the speed limit did not affect our conclusions. Changing the speed limit does change the number of clusterhead changes for the other algorithms, but our traffic flow algorithm maintained almost 0 changes for any of the speed limits we tested.

We wanted to verify that our algorithm would choose the appropriate clusterhead for the majority of traffic, not just those vehicles that are going straight. So, we tested our algorithm at an additional intersection of 4 lanes, where 2 lanes are turning left and 2 lanes are going straight. We changed the traffic rate between the two directions (left and straight) to $60 \%$ and $40 \%$, respectively. The results for our algorithm show that the clusterhead was always selected from one of the lanes where the traffic rate was $60 \%$. So, our algorithm does select the clusterhead from the lane that follow the direction of most of the traffic.

\section{CONCLUSION}

We presented an algorithm for clusterhead selection based on the traffic flow of vehicles in the cluster. With the availability of lane detection, lane direction and map matching, we were able to select the most stable clusterhead. We tested our algorithm using real life scenarios and followed the selected clusterhead through two intersections. Our algorithm showed longer clusterhead lifetime than Lowest-ID, Highest-Degree and the Utility Function algorithms. Our future work will be to apply our algorithm to inter-cluster communication algorithms.

\section{REFERENCES}

[1] H. Arbabi and M. C. Weigle. Highway mobility and vehicular ad-hoc networks in ns-3. In Proceedings of the Winter Simulation Conference, Baltimore, MD, Dec 2010.

[2] R. Aufrere, R. Chapuis, and F. Chausse. A model-driven approach for real-time road recognition. Machine Vision and Applications, 13(2 2001):95-107, 2001.

[3] M. Bertozzi and A. Broggi. Gold: A parallel real-time stereo vision system for generic obstacle and lane detection. IEEE Transactions on Image Processing, 7(1):62-81, January 1998.

[4] A. J. Dean and S. N. Brennan. Terrain-based road vehicle localization on multi-lane highways. In Proceedings of the American Control Conference, pages 707-712, Piscataway, NJ, USA, 2009. IEEE Press.

[5] P. Fan, J. G. Haran, J. Dillenburg, and P. C. Nelson. Traffic model for clustering algorithm in vehicular ad-hoc network. In Proceedings of the Consumer Communications and Networking Conference, volume 1, pages 168-172, 2006.

[6] P. Fan, J. G. Haran, J. F. Dillenburg, and P. C. Nelson. Cluster-based framework in vehicular ad-hoc networks. In $A D H O C-N O W$, pages 32 42, 2005.

[7] M. Garg and R. K. Shyamasundar. A distributed clustering framework in mobile ad hoc networks. In Proceedings of the International Conference on Wireless Networks, pages 32-38, 2004.

[8] T. R. Henderson, S. Roy, S. Floyd, and G. F. Riley. ns-3 project goals. In Proceeding from the 2006 workshop on ns-2: the IP network simulator, 2006.

[9] W. Holfelder. Vehicle safety communications in the US. In Proceedings of the 2007 ITS World Congress, London, U.K., 2006. 
[10] M. Jabbour, P. Bonnifait, and V. Cherfaoui. Enhanced local maps in a GIS for a precise localisation in urban areas. In Proceedings of the 9 th IEEE Conference on Intelligent Transportation Systems (ITSC 2006), 2006.

[11] M. Jiang, J. Li, and Y. Tay. Cluster based routing protocol. Internet Draft. draft-ietf-manet-cbrp-spec-01, August 1999.

[12] S. Kuklinski and G. Wolny. Density based clustering algorithm for VANETs. Testbeds and Research Infrastructures for the Development of Networks and Communities, International Conference on, 0:1-6, 2009.

[13] C. D. McGillem and T. S. Rappaport. A beacon navigation method for autonomous vehicles. IEEE Transactions on Vehicular Technology, 38(3):132-139, 1989.

[14] NAVTEQ. NAVTEQ's NAVSTREETS Street Data Reference Manual, 3.4 edition, October 2009.

[15] T. Oskiper, Z. Zhu, S. Samarasekera, and R. Kumar. Visual odometry system using multiple stereo cameras and inertial measurement unit. In Proceedings Computer Vision and Pattern Recognition, 2007.

[16] A. Ramalingam, S. Subramani, and K. Perumalsamy. Associativity based cluster formation and cluster management in ad hoc networks. In
Proceedings of the 9th International Conference On High Performance Computing, Bangalore, India, December 2002.

[17] M. Treiber and D. Helbing. Realistische mikrosimulation von straßenverkehr mit einem einfachen modell. In Proceedings of the 16th Symposium "Simulationstechnik ASIM 2002" Rostock, pages 514-520, September 2002.

[18] M. Treiber, A. Hennecke, and D. Helbing. Congested traffic states in empirical observations and microscopic simulations. Phys Rev E Stat Phys Plasmas Fluids Relat Interdiscip Topics, 62(2 Pt A):1805-24, 2000.

[19] US Department of Transportation. Standard Specification for Telecommunications and Information Exchange Between Roadside and Vehicle Systems - 5 GHz Band Dedicated Short Range Communications (DSRC) Medium Access Control (MAC) and Physical Layer (PHY) Specifications. ASTM E2213-03, August 2003.

[20] G. Wolny. Modified DMAC clustering algorithm for VANETs. In ICSNC '08: Proceedings of the 2008 Third International Conference on Systems and Networks Communications, pages 268-273, Washington, DC, USA, 2008. IEEE Computer Society. 